\title{
Primer registro de Munidopsis cubensis Chace, 1942 (Crustacea: Anomura: Munidopsidae) para el mar Caribe sur, Colombia
}

\author{
Yeison Herrera-Medina, Adriana Bermúdez-Tobón, Gabriel R. Navas-Suárez* \\ Programa de Biología, Facultad de Ciencias Exactas y Naturales, Universidad de Cartagena, Cartagena, Colombia
}

\begin{abstract}
Resumen
Se registra la especie Munidopsis cubensis para el mar Caribe sur, Colombia, a partir de un espécimen recolectado con red de arrastre en noviembre de 2009, costa afuera de Punta Canoa (Bolívar), a $776 \mathrm{~m}$ de profundidad. Se presentan los caracteres diagnósticos de la especie y se añade información sobre su distribución y ámbitos batimétrico y geográfico. Con este nuevo registro se aumenta a 24 el número de especies de Munidopsis registradas para Colombia.
\end{abstract}

Palabras clave: Munidopsis cubensis, Caribe, Colombia, Decapoda.

First record of Munidopsis cubensis Chace, 1942 (Crustacea: Anomura: Munidopsidae) for the Southern Caribbean Sea, Colombia.

\begin{abstract}
Munidopsis cubensis is reported for the first time for the Southern Caribbean Sea, Colombia. The specimen was collected in November 2009, using a trawl, offshore of Punta Canoa (Bolívar) at a depth of $776 \mathrm{~m}$. We present the species diagnostic characters, as well as its distribution and bathymetric and geographic information. This record increases to 24 the number of known species of Munidopsis in Colombia.
\end{abstract}

Key words: Munidopsis cubensis, Caribbean, Colombia, Decapoda.

\section{Introducción}

En la última década la exploración de las plataformas y taludes ubicados en aguas colombianas ha cobrado gran interés debido al desconocimiento de la biota que allí habita y del impacto a que se verá sometida debido al incremento de actividades como la pesca de arrastre de profundidad y la prospección minera (Navas, et al., 2010).

Entre los componentes megabentónicos de la plataforma y el talud del Caribe colombiano, los crustáceos decápodos de la superfamilia Galatheoidea Samouelle, 1819, son un grupo conspicuo por su riqueza y abundancia (Navas, et al., 2003). Esta superfamilia incluye las familias Galatheidae Samouelle, 1819, Porcellanidae Harwort, 1825, Munididae (Ahyong, et al., 2010) y Munidopsidae Ortmann, 1898 (Macpherson \& Baba, 2011). La ausencia del flagelo en el exópodo del primer maxilípedo es el principal carácter que diferencia a los Munidopsidae del resto de los Galatheoidea (Ahyong, et al., 2010).

Munidopsidae es un grupo de Galatheoidea de taludes y mar profundo, en donde habita en una gran variedad de ambientes, incluidos respiraderos hidrotermales $\mathrm{y}$ surgencias frías (Chevaldonne \& Olu, 1996). A diferencia de muchas de las especies de Munida, presentes en gran abundancia en los fondos blandos de la plataforma y el talud superior, Munidopsis presenta menores densidades y una estrategia reproductiva que se diferencia de la de Munida por la menor cantidad de huevos de un tamaño mucho mayor (Wenner, 1982).

Los géneros que componen la familia Munidopsidae son: Galacantha A. Milne Edwards, 1880, Leiogalathea Baba, 1969, Shinkaia Baba y Williams, 1998 y Munidopsis Whiteaves, 1874 (Macpherson \& Baba, 2011). Los especímenes pertenecientes al género Munidopsis se reconocen por tener el rostro triangular o en forma de espina, así como segmentos abdominales con surcos y estrías transversales, con o sin espinas; sexto segmento con lóbulos laterales frecuentemente bien pronunciados, margen medio transverso o convexo, ocasionalmente

\footnotetext{
*Correspondencia:

Gabriel R. Navas-Suárez, gnavass@unicartagena.edu.co

Recibido: 29 de enero de 2014

Aceptado: 27 de abril de 2014
} 
pronunciado posteriormente y que sobrepasa los lóbulos laterales; subdivisiones del telson completas; flagelo antenal que usualmente sobrepasa el primer pereiópodo; ausencia de flagelo en el primer maxilípedo; epipoditos presentes en el segundo y tercer maxilípedos, algunas veces en el primer pereiópodo e, incluso, sobre el segundo y tercero; dáctilos de los pereiópodos segundo a cuarto con el margen flexor entero o con una línea de espinas fijas que disminuye de tamaño a partir de la parte proximal hacia el final de la articulación, casi nunca subquelado, con la parte distal del própodo y primer y segundo gonópodos presentes (Baba, et al., 2009).

Las especies del género Munidopsis suelen habitar en fondos blandos de los taludes continentales, en las llanuras abisales y también se han encontrado en las fosas hidrotermales y manantiales fríos de profundidad de todo el mundo (Baba, 2005; Baba, et al., 2008; Macpherson \& Segonzac, 2005). En el océano Atlántico se han descrito más de 70 especies, 23 de ellas presentes en aguas colombianas, incluida la recientemente registrada Munidopsis robusta (Fierro, et al., 2008; Ortega-Echeverría et al., 2013).

Este registro de $M$. cubensis Chace, 1942, complementa el conocimiento morfológico de la especie, aporta nueva información sobre su ámbito geográfico, sobre las características específicas de su hábitat y sobre las relaciones faunísticas entre las plataformas y taludes de las ecorregiones del Gran Caribe, y nutre el Inventario Nacional de Biodiversidad.

\section{Materiales y métodos}

El espécimen estudiado fue recolectado a bordo del buque B/I Ancón, costa afuera de Punta Canoa (10 34' 43.9" N y $75^{\circ} 39^{\prime}$ 52,9” O) en el departamento de Bolívar, el 16 de noviembre de 2009 a $776 \mathrm{~m}$ de profundidad. La captura se realizó mediante arrastre de 20 minutos contados a partir del momento en que tocaba fondo y a una velocidad aproximada $\mathrm{de} 5,6 \mathrm{~km} / \mathrm{h}$. La recolecta se realizó con una red de arrastre (tipo semiglobo) diseñada para el muestreo a estas profundidades; dicha red contaba con una relinga superior de 7,70 $\mathrm{m}$ y una inferior de 9,50 m, un copo con ojo de malla de $30 \mathrm{~mm}$ y un sobrecopo de paño sin nudos y ojo de malla de $10 \mathrm{~mm}$; la red estaba dotada de dos compuertas metálicas de 91 x $63 \mathrm{~cm}$. Después de su captura, el organismo se enjuagó con agua de mar y se preservó en etanol al 70 \%. Las características oceanográficas de la columna de agua se evaluaron con una sonda multiparamétrica CTDO (General Oceanics Ocean Seven 316 plus) y los sedimentos se recolectaron por medio de un nucleador de caja Gomex GM-2525-II (25 x $25 \mathrm{~cm})$ y luego se analizó su composición granulométrica. El ejemplar se identificó por medio de estereoscopio empleando los trabajos de Chace, 1942 y Mayo, 1974, y se depositó en el Museo de Historia Natural Marina de Colombia en Santa Marta (MHNMC).

\section{Resultados y discusión}

\section{Munidopsis cubensis Chace, 1942}

La figura 1 ilustra el organismo en su vista dorsal y ventral.

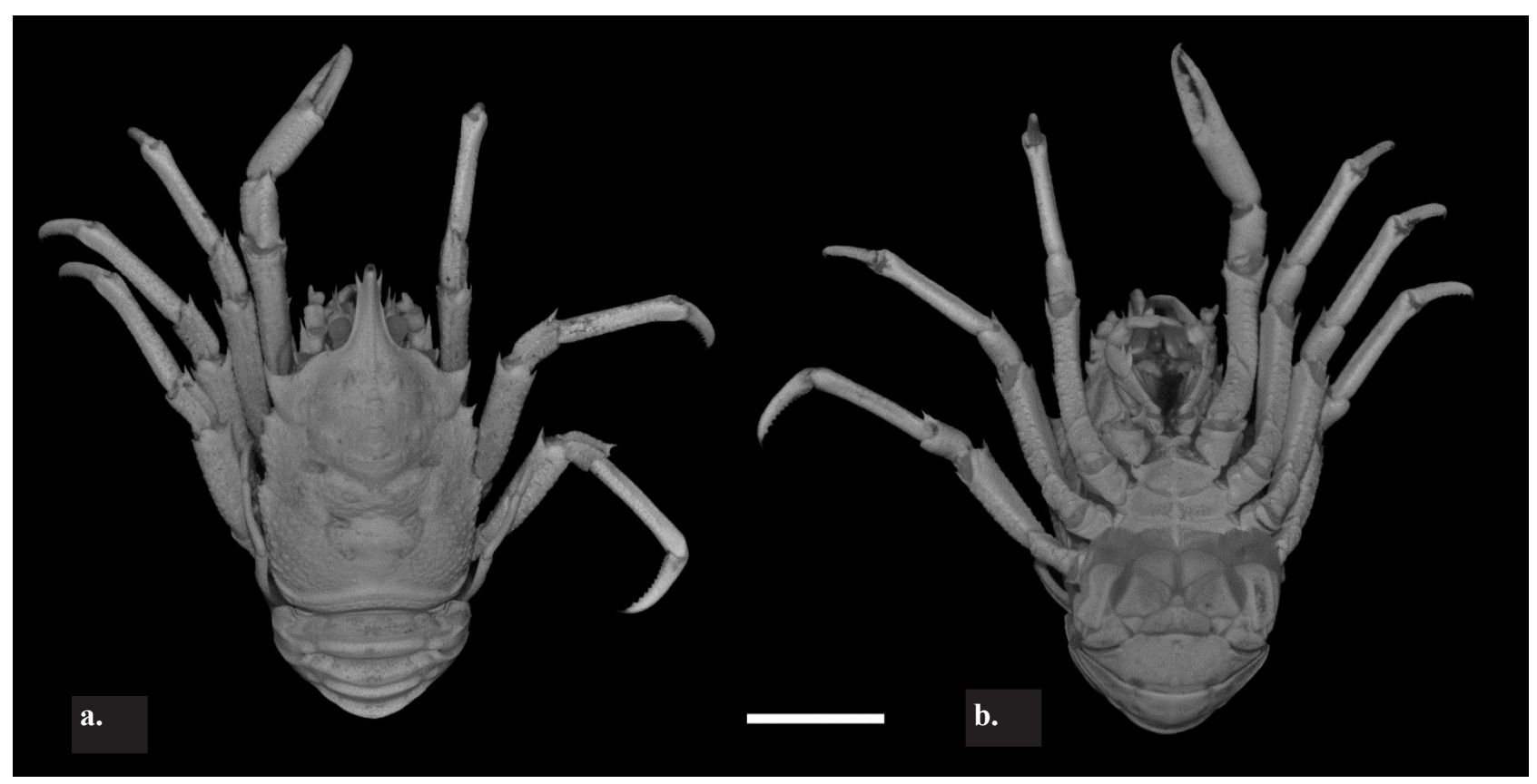

Figura 1. Munidopsis cubensis. a. Vista dorsal, b. Vista ventral. La escala corresponde a $1 \mathrm{~cm}$. 


\section{Referencias}

Chace, 1942: 78-80, figs. 27 y 28, 72 (clave); Pequegnat \& Pequegnat, 1970: 138 (clave); Pequegnat \& Pequegnat, 1971: 4 (clave); Mayo, 1974, 125-132, figs. 16 y 17, 34 (clave); Fierro, et al., 2008: 7 (lista); Baba, et al., 2008, 138.

\section{Material examinado}

Ecorregión del Caribe Oceánico (Colombia), costa afuera de Punta Canoa, departamento de Bolívar. Expedición Invemar-ANH II (estación 285, $10^{\circ} 34^{\prime} 43,9^{\prime \prime} \mathrm{N}, 75^{\circ}$ 39' 52,9" O), $776 \mathrm{~m}$ de profundidad, 16 de noviembre, 2009, 1 ऊ̂ (MHNMC-8282), longitud del caparazón desde la base del rostro: $18,3 \mathrm{~mm}$, ancho máximo del caparazón: $15,4 \mathrm{~mm}$.

\section{Diagnosis}

Rostro levantado, armado en el extremo con un par de espinas laterales. Región gástrica anterior del caparazón con un par de espinas epigástricas mediales. Margen frontal desarmado. Segundo y tercer segmentos abdominales armados cada uno con una espina media sobre la cresta anterior, cuarto segmento con tubérculo medial no espinoso (Mayo, 1974).

\section{Descripción}

Esta se complementa con las dadas por Chace, 1942 y Mayo, 1974. Caparazón más largo que ancho, abovedado transversalmente. Región gástrica levemente inflada, armada con un par de espinas anteriores distinguibles, superficie dorsal rugosa y granulada transversalmente, surco gástrico con un dentículo central pequeño. Surco cervical visible detrás de la región gástrica; la parte media del surco cervical es más profunda que sus ramas posteriores; bordes posteriores de los surcos aserrados pero sin espinas. Cerdas cortas y curvas, la mayoría sobre la superficie dorsal expuesta. Rostro carinado. Longitud del rostro de aproximadamente la mitad de la del caparazón; la parte distal doblada hacia arriba forma un ángulo de aproximadamente $45^{\circ}$ desde la horizontal, armado con un par de espinas en el extremo; porción horizontal amplia. Abdomen armado con una espina media sobre la cresta transversal anterior del segundo y tercer segmento abdominal; una pequeña espina sobre el margen posterior del segundo somite abdominal; cuarto segmento abdominal con una pequeña espina media sobre el margen anterior; quinto y sexto segmentos relativamente lisos. Esternón desarmado y suave, pero con crestas entre segmentos altas y agudas. Ojos incoloros, desarmados y móviles; diámetro de las córneas ligeramente más ancho que los pedúnculos oculares. Segmento basal del pedúnculo antenular hinchado; dos espinas distolaterales, la más distal, ligeramente más ancha. Segmento basal de la antena ancho, con un diente triangular expandido de forma ventromedial; segundo segmento con una espina distolateral puntiaguda; tercer y cuarto segmentos desarmados, aunque el cuarto presenta un lóbulo distal. Tercer maxilípedo con una espina distolateral sobre el mero y con dos espinas grandes ventromediales. Quelípedos de aproximadamente la mitad del caparazón; pereiópodos gruesos y rugosos, con espinas terminales sobre las articulaciones meral y carpal.

\section{Observaciones}

Según la clave de Mayo, 1974, M. cubensis no presenta espinas sobre el margen del borde posterior del caparazón; sin embargo, en la descripción dada en este mismo trabajo se menciona la presencia de dos pequeñas espinas, las cuales fueron descritas por Chace, 1942, como diminutas e insignificantes, lo que sugiere el poco valor taxonómico de este carácter. El ejemplar recolectado en aguas colombianas no presenta espinas, espínulas, tubérculos ni protuberancias en el margen posterior del caparazón, así como tampoco el quelípedo derecho.

Para Chace, 1942, M. cubensis presenta el rostro armado lateralmente y curvado hacia arriba de forma semejante a los de Munidopsis expansa Benedict, 1902, y Munidopsis gilli Benedict, 1902, pero se diferencia de ambas por tener solamente un par de espinas gástricas bien desarrolladas sobre el caparazón. M. gilli tiene dos tubérculos conspicuos, línea espinosa lateral menos desarrollada, el caparazón menos ornamentado y un diente sobre la parte media del cuarto terguito abdominal. $M$. expansa presenta el caparazón más ornamentado, no tiene espinas gástricas ni abdominales, y tampoco epípodos sobre los terceros pereiópodos (Mayo, 1974). De las especies presentes en aguas colombianas, la más cercana morfológicamente es Munidopsis bradleyi Pequegnat \& Pequegnat, 1971, la cual se diferencia de M. cubensis por tener el rostro menos curvado, epípodos solamente sobre los quelípedos, más espinas gástricas y espinas abdominales pareadas.

\section{Hábitat}

Los especímenes se capturaron en la capa de agua intermedia antártica, la cual se ubica entre los 300 y $800 \mathrm{~m}$ de profundidad, con niveles mínimos de salinidad cercanos a 34,7 y temperatura de $6,5^{\circ} \mathrm{C}$ (Lozano-Duque, et al., 2010). A la profundidad de captura se presentó una temperatura de $6,5^{\circ} \mathrm{C}$, salinidad de 34,8 , saturación de $\mathrm{O}_{2}$ de $56,9 \%$ y pH de 8,2 . El sedimento estaba conformado principalmente por limo fino y lodo, con un porcentaje de carbonato de 4,9 y $13,7 \%$ de materia orgánica.

\section{Distribución y ámbito batimétrico}

La especie fue descrita por Chace, 1942, a partir de un macho capturado en 1939 al noreste de Puerto Cayo Moa, provincia de Oriente, Cuba $\left(20^{\circ} 46^{\prime} \mathrm{N}, 74^{\circ} 59^{\prime} \mathrm{O}\right)$, 
a $1.144 \mathrm{~m}$ de profundidad, y su distribución se extendió hasta el Estrecho de La Florida con una hembra capturada allí $\left(24^{\circ} 02^{\prime} \mathrm{N}, 83^{\circ} 02^{\prime \prime} \mathrm{O}\right)$ a una profundidad entre 869 y 759 m (Mayo, 1974). En la base de datos del Museo de Historia Natural del Instituto Smithsoniano (NMNH, http://collections.mnh.si.edu/search/iz/, 2014) se registra una hembra capturada por el $R / V$ Miguel Oliver en 2011 frente a Nicaragua $\left(11^{\circ} 31^{\prime} 54,3\right.$ " N, $\left.83^{\circ} 8^{\prime} 28,86^{\prime \prime} \mathrm{O}\right)$, a una profundidad entre 791 y $861 \mathrm{~m}$.

Teniendo en cuenta estos registros, y el ejemplar capturado en aguas colombianas, M. cubensis se puede considerar como una especie bentónica que habita sobre fondos blandos de la plataforma y el mar profundo a una profundidad conocida de 759 a $1.144 \mathrm{~m}$. Su distribución abarca las ecorregiones de las Antillas Mayores, el sur del Golfo de México y el Caribe suroccidental (Figura 2), y es congruente con la de las seis especies colombianas con distribución restringida al mar Caribe: Munidopsis brevimana, $M$. colombiana, $M$. ramahtaylorae, $M$. reynoldsi, $M$. riveroi y $M$. spinoculata, las cuales se encuentran desde los 134 a los $4.151 \mathrm{~m}$ de profundidad (Navas, et al., 2013). Estas especies están restringidas a la provincia del Atlántico Noroeste Tropical (Spalding, et al., 2007), provincia con aguas someras cálidas aportadas por la corriente de las Antillas en la parte externa del Arco Antillano, por la Corriente del Caribe, que atraviesa el mar
Caribe hasta el Golfo de México, por la corriente de La Florida y por el giro Panamá-Colombia (Lozano-Duque, et al., 2010); este último podría transportar los organismos durante las fases larvales hacia el sur del Caribe colombiano (Bermúdez, et al., 2008).

Los ejemplares de $M$. cubensis tienen poca representación en las capturas en el Caribe (Mayo, 1974); esta menor abundancia de las especies de Munidopsis comparada con la de las especies de la familia Munididae (Navas, et al., 2012) fue atribuida por Mayo, 1974, y por Creasey, et al., 2000, a una probable tendencia a vivir en hábitats más restringidos y a preferir las oquedades o, al menos, a vivir semienterradas en el sedimento. Wenner, 1982, determinó que las especies de Munidopsis tienden a enterrarse en el sustrato ante la presencia de las artes de muestreo.

\section{Agradecimientos}

Los autores agradecen a la doctora Diana Marcela Bolaños Rodríguez, docente del programa de Biología de la Universidad de Cartagena, por la revisión del manuscrito; a la bióloga Natalia Rodríguez, por su apoyo en la recolección y separación del material biológico durante las expediciones de campo.

\section{Conflicto de interés}

Los autores declaran que no tienen ningún conflicto de interés.

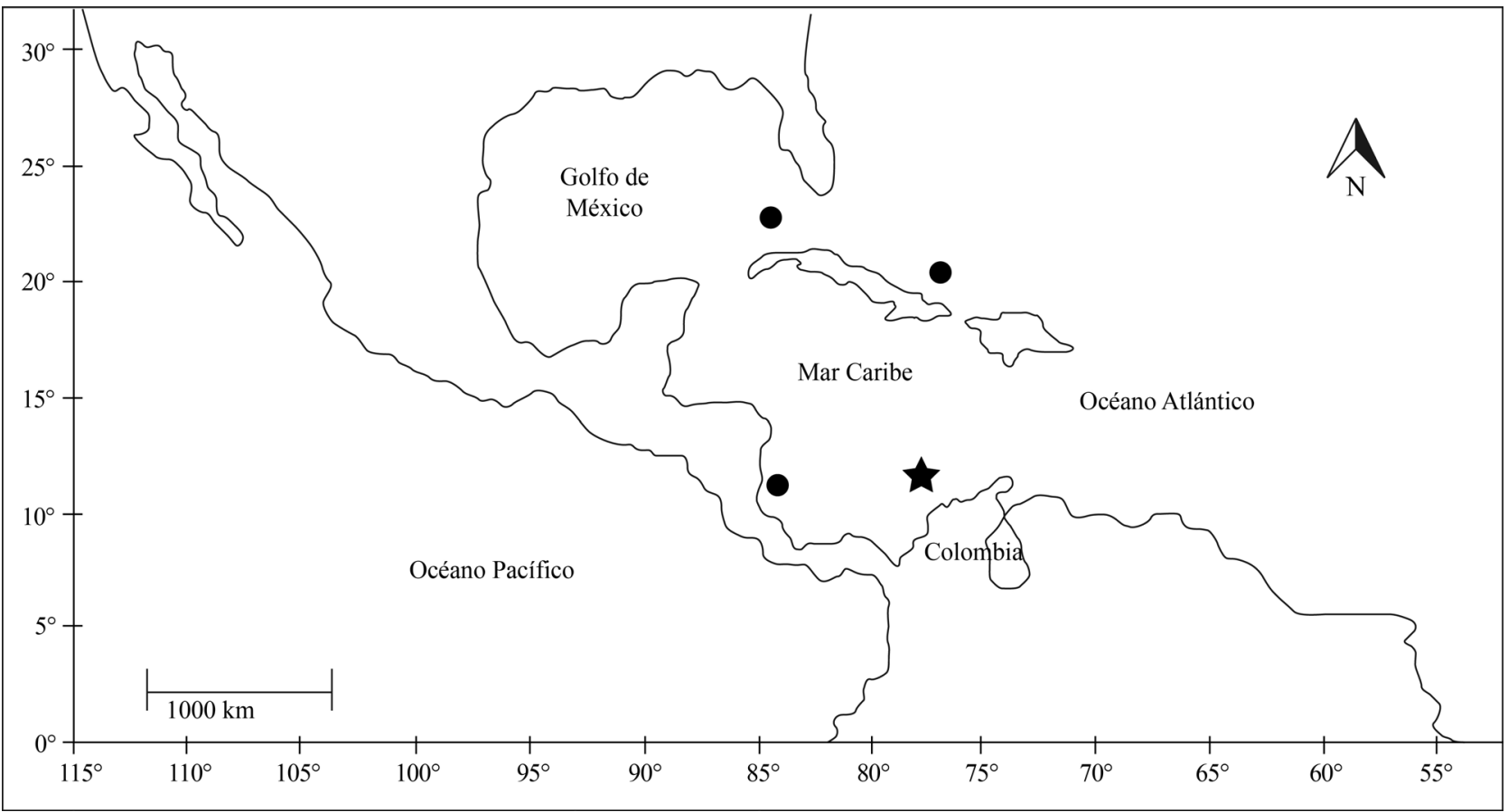

Figura 2. Mapa de la distribución de Munidopsis cubensis. El nuevo registro en aguas colombianas está representado por una estrella negra; los registros previos se representan con círculos negros (Chace, 1942; Mayo, 1974; NMNH, 2014). 


\section{Bibliografía}

Ahyong, S.T., Baba, K., Macpherson, E., Poore, G. 2010. A new classification of the Galatheoidea (Crustacea: Decapoda: Anomura), Zootaxa 2676(1):57-68.

Baba, K. 2005. Deep-sea chirostylid and galatheid crustaceans (Decapoda: Anomura) from the Indo-West Pacific, with a list of species. Galathea Reports 20:1-317.

Baba, K., Macpherson, E., Lin. C-W, Chan, T-Y. 2009. Crustacean Fauna of Taiwan: Squat Lobsters (Chirostylidae and Galatheidae). National Taiwan Ocean University, Keelung. 311 pp.

Baba, K., Macpherson, E., Poore, G.C.B., Ahyong, S.T., Bermúdez, A., Cabezas, P., Lin, C.W., Nizinski, M., Rodrigues, C., Schnabel, K.E. 2008. Catalogue of squat lobsters of the world (Crustacea: Decapoda: Anomura families Chirostylidae, Galatheidae and Kiwaidae), Zootaxa 1905:1-220.

Bermúdez, A., Navas, G.R., Campos, N.H. 2008. Populationsstruktur und genetische Isolierung von drei Springkrebs-Arten. Der Einfluss der ozeanographischen Merkmale und der geographischen Distanz zwischen den Populationen entlang der karibischen Küste von Kolumbien. VDM Verlag, Saarbrücken. 116 pp.

Chace, F.A. 1942. Reports on the scientific results of the Atlantis expeditions to the West Indies, under the joint auspices of the University of Havana and Harvard University, the Anomuran Crustacea. I Galatheidea. Torreia 11:1-106.

Chevaldoneé, P., Olu, K. 1996. Occurrence of anomuran crabs (Crustacea: Decapoda) in hydro thermal vent and coldseep communities: A review, Proceedings of the Biological Society of Washington, 109(2):286-298.

Creasey, S.A., Rogers, P.T., Gage, J., Jollivet, D. 2000. Genetic and morphometric comparisons of squat lobster, Munidopsis scobina (Decapoda: Anomura: Galatheidae) populations, with notes on the phylogeny of the genus Munidopsis. Deep-Sea Research II 47:87-118.

Fierro, M., Navas, G.R., Bermúdez, A., Campos, N.H. 2008. Lista de chequeo de las familias Galatheidae y Chirostylidae (Crustacea: Decapoda: Anomura) del neotrópico. Biota Colombiana, 9(1):1-20.

Macpherson, E., Baba K. 2011. Taxonomy of squat lobsters. En: G.C.B. Poore, S.T. Ahyong, J. Taylor (editores), The Biology of Squat Lobsters. Csiro Publishing, Australia. 39-72 pp.

Macpherson, E., Segonzac, M. 2005. Species of the genus Munidopsis (Crustacea, Decapoda, Galatheidae) from the deep Atlantic Ocean, including cold-seep and hydrothermal vent areas. Zootaxa 1095:1-60.

Mayo, B.S. 1974. The systematics and distribution of the deep-sea genus Munidopsis (crustacea, Galatheidae) in the Western Atlantic Ocean. Tesis de Doctorado, Universidad de Miami, Coral Gables, Florida, 433 pp.
Lozano-Duque, Y., Medellín-Mora, J., Navas, G.R. 2010. Contexto climatológico y oceanográfico del mar Caribe colombiano. En: G.R. Navas, C. Segura-Quintero, M. Garrido-Linares, M. Benavides-Serrato y D. Alonso (editores), Biodiversidad del margen continental del Caribe colombiano, Serie de publicaciones especiales, Invemar, Santa Marta, número 20, 53-84 pp.

Navas, G.R., Bermúdez, A., Cruz, N., Campos, N.H. 2003. Galatéideos (Decapoda, Anomura, Galatheidae) del Caribe colombiano, incluyendo doce primeros registros. Boletín de Investigaciones Marinas y Costeras 32:181-214.

Navas, G.R., Segura-Quintero, C., Garrido-Linares, M., Benavides-Serrato, M., Alonso, D. 2010. Biodiversidad del margen continental del Caribe colombiano. Serie de publicaciones especiales, Invemar, Santa Marta, número 20, $458 \mathrm{pp}$.

Navas, G.R., Bermúdez, A., Ángel-Yunda, C., Campos, N.H. 2012. Composición y distribución de los ensamblajes de crustáceos galateoideos (Decapoda: Anomura) presentes en aguas colombianas. Caldasia 34(2):367-384 .

Navas, G.R., Bermúdez, A., Campos N.H., Ángel-Yunda, C. 2013. Afinidades biogeográficas de los galateoideos (Decapoda: Anomura) del Caribe y Pacífico colombiano. Revista MVZ Córdoba 18(2):3501-3511

NMNH. 2014. Munidopsis cubensis. Smithsonian National Museum of Natural History. http://collections.mnh.si.edu/ search/iz/, 28.01.2014.

Ortega-Echeverría, C., Bermúdez, A., Navas, G.R. 2013. Presencia de Munidopsis robusta en el Caribe colombiano (A. Milne Edwards, 1880) (Crustacea: Anomura: Munidopsidae), Universitas Scientiarum 19(1):43-49.

Pequegnat, L.H., Pequegnat, W.E. 1970. Deep-sea Anomurans of Superfamily Galatheoidea with Descriptions of Three New Species. En: W.E. Pequegnat, F.A. Chace (editores), Contributions on the Biology of the Gulf of Mexico, Texas A \& M University, 125-170 pp.

Pequegnat, W.E., Pequegnat, L.H. 1971. New species and new records of Munidopsis (Decapoda: Galatheidae) from the Gulf of Mexico and Caribbean Sea. Supplement to Texas A \& M University Oceanographic Studies, vol. 1, Gulf Publishing Co., Houston, 25 pp.

Spalding, M.D., Fox, H.E., Allen, G.R., Davison, N., Ferdaña, Z.A., Finlayson, M., Halpern, B.S., Jorge, M.A., Lombana, A., Lourie, S.A., Martin, K.D., McManus, E., Molnar, J., Recchia, C.A., Robertson, J. 2007. Marine Ecoregions of the World: A Bioregionalization of Coastal and Shelf Areas. BioScience 57(7):573-583.

Wenner, E.L. 1982. Notes on the distribution and biology of Galatheidae and Chirostylidae (Decapoda: Anomura) from the Middle Atlantic Bight. Journal of Crustacean Biology 2(3):360-377. 